\title{
Vascular Care Services during the Covid-19 Pandemic in a South Asian Country: Implications for Limb Salvage
}

\author{
Bashar AHM* \\ Department of Vascular Surgery, National Institute of \\ Cardiovascular Diseases and Hospital, Dhaka, Bangladesh \\ *Corresponding author: Abul Hasan Muhammad \\ Bashar, Department of Vascular Surgery, National \\ Institute of Cardiovascular Diseases and Hospital, Dhaka, \\ Bangladesh
}

Received: August 09, 2021; Accepted: October 06, 2021; Published: October 13, 2021

\begin{abstract}
Objective: Covid-19 continues its negative impact on limb salvage in patients with limb ischemia and vascular trauma. Bangladesh fought the $1^{\text {st }}$ and $2^{\text {nd }}$ waves of the Covid-19 pandemic during April through August, 2020 and in April-May, 2021, respectively. Infections have been rising once again since early June 2021 to reach a positivity rate over $30 \%$. With limited resources, vascular surgical care during the pandemic has been an unprecedented challenge for a developing country like Bangladesh. As a small vascular community, we have adopted a strategy focused on conserving essential resources including manpower while delivering essential vascular care. The present study evaluates the effectiveness of our pandemic-time guideline-based working strategy with a focus on limb salvage.
\end{abstract}

Methods: Essential vascular services were provided by customized teams throughout the Covid-19 pandemic at two referral centers according to our working guidelines. Data were analyzed and key limb salvage outcomes compared between the two pandemic waves by chi-square test.

Results: A total of 1792 vascular surgical cases were addressed during the pandemic- over $90 \%$ of which involved limb or life-saving procedures. Overall mortality was $0.6 \%$ and overall limb salvage rate for acute and Chronic Limb Threatening Ischemia (CLTI) were $95.3 \%$ and $76.8 \%$, respectively. Limb salvage rate for CLTI was significantly higher in the $2^{\text {nd }}$ wave compared with the $1^{\text {st }}(P<0.05)$. Rate of Covid-19 infection among vascular workforce was about $20 \%$.

Conclusion: Emergency-focused altered working guideline has produced satisfactory results in terms of continuing emergency vascular services, improving limb salvage and safeguarding vascular workforce during Covid-19 pandemic in a developing country. Conservation of resources including manpower remains relevant in the context of a highly probable $3^{\text {rd }}$ wave of infection.

Keywords: Covid-19; Limb salvage; Third wave; Vascular emergency services; Health care workers; Customized team approach; Bangladesh

\section{Introduction}

Severe Acute Respiratory Syndrome (SARS) corona virus disease or Covid-19 situation continues to linger worldwide. While the pandemic has been brought under control in Europe and North America, it prevails at considerable intensity in South America and Asia [1]. A dangerous second wave recently swept across the Indian subcontinent, particularly India which remained the center of attention of the pandemic world for the most part of May, 2021 as she recorded more than 3,00,000 new cases and over 4000 deaths every day [2,3]. With over 30 million cases and 4,00,000 deaths, India has been one of the worst to suffer from the pandemic. Bangladesh is a small and densely populated developing country of the Indian subcontinent [4] where the pattern of Covid-19 infection had been somewhat different from the rest of the subcontinent. The maximum intensity of the disease in Bangladesh was in June through August, 2020 with a test vs case ratio of around $25 \%$. New infection and death subsided toward the end of the year 2020. Just when everything appeared under control, the $2^{\text {nd }}$ wave started to become apparent with the beginning of the summer. Test vs case ratio which had fallen below 3\% jumped to more than 20\% in the early weeks of April 2021 [5]. Covid treatment facilities including Intensive Care Units (ICU) once again became full to their capacities within a short span of time. Though a downward trend was achieved in late May, 2021, infection and death have once again started to rise since the beginning of June (Figure 1). Experts believe that Bangladesh is now going through a $3^{\text {rd }}$ wave of the pandemic which has already proved far worse than the previous waves as the demand for essential treatment resources including ICU and oxygen continues to soar.

Surgical patient care during Covid-19 had dramatically changed all over the world with most disciplines adopting a drastically modified working protocol. Providing only the very essential services had been the mainstay of such modified approach [6,7]. However, with improving conditions, healthcare system in many countries did attempt a watchful return to normalcy. This was particularly true for European countries [8-10]. Vascular surgery is a relatively small but rapidly flourishing discipline in Bangladesh. The impact of Covid-19 


\section{Covid-19 Positivity Rate (Test vs Case)}

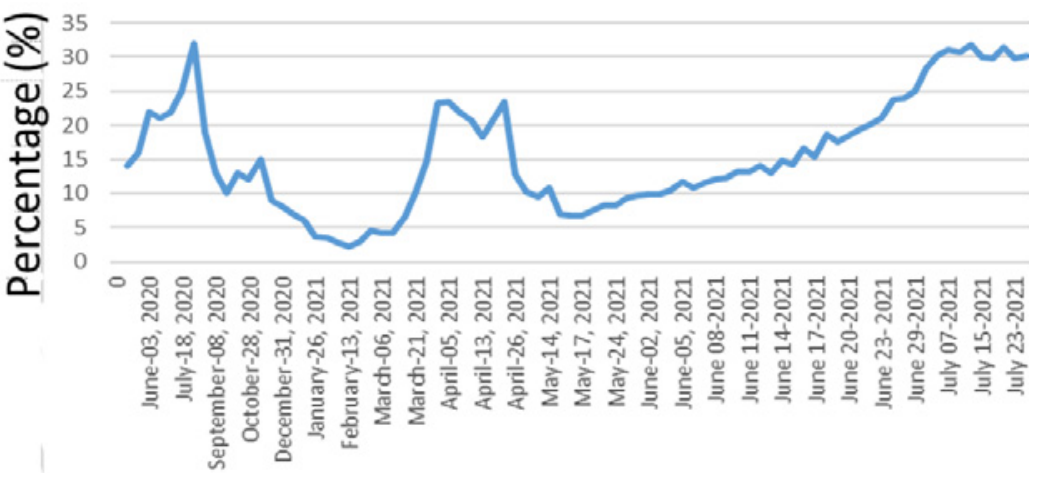

Figure 1: Trend in positivity rate of Covid-19 infection in Bangladesh throughout the pandemic.

on vascular care in the country has been quite profound. With limited number of dedicated vascular surgeons against a large population, vascular surgical care in Bangladesh during Covid-19 has been facing unprecedented challenges. The challenges were initially met with a strategy of rendering only the essential services postponing all routine issues based on our own working guideline [11]. Vascular routine ward remained closed for the most part of the $1^{\text {st }}$ and $2^{\text {nd }}$ waves of the pandemic, so were the routine operation theatres. These practices were basically in line with those being practiced elsewhere in the world $[12,13]$. Indeed, several proposed vascular guidelines recommended such streamlined practices during the pandemic [14]. However, with improving Covid situation, vascular routine works resumed and reached the pre-pandemic volume late last year.

In Bangladesh, like all other disciplines of healthcare, the delivery of vascular surgical care has two separate avenues- public and private. In the public sector, National Institute of Cardiovascular Diseases \& Hospital (NICVD) is the largest facility that offers emergency vascular care along with all other routine vascular activities. In recent years, Bangladesh Specialized Hospital Limited (BSHL), Dhaka has emerged as a center of excellence in the private sector where the basic infrastructure for a comprehensive vascular care is available. Together, these two centers have shared most of the vascular emergency workload of the country during the Covid-19 pandemic.

The community of Health care workers (HCW) has been one of the worst to suffer from Covid-19 all over the world [15-19]. US Center for Disease Control (CDC) reported 106976 cases and 560 deaths among US HCW last year [16,17]. In Italy, more than 100 doctors had died out of a total Covid mortality of over 34000 [18]. According to the data recently released by the Indian Medical Association (IMA), India has also lost a staggering number of 747 doctors to Covid-19 [19]. In Bangladesh, over the last 16 months, more than 220 doctors lost their lives due to the virus out of a total mortality of 21,000 , which is an alarmingly high number. Not only that the rate of infection has been disproportionately higher among the HCW, case fatality rate of nearly $4 \%$ among doctors of Bangladesh has also been the highest in the world [20]. Therefore, protecting the HCW, particularly doctors is a logical priority in the healthcare policy during the pandemic. The vascular surgical community of Bangladesh has been pursuing the specific objectives of protecting manpower and conserving resources while rendering essential services. In this study, we analyzed our experiences throughout the Covid-19 pandemic at two high volume vascular care centers and evaluated the effectiveness of our pandemic time guideline-based strategy in achieving these objectives.

\section{Methods}

The study was approved by the Ethical Review Board of NICVD and Institutional Review Board of BSHL. Customized vascular surgical teams were formed on the basis of the following criteria; involving the barest minimum manpower for a given task with utilization of younger members as much as possible, involving senior manpower mostly in the decision making process and not physically involving members aged 50 years or older and those with preexisting conditions as much as possible.

Essential vascular services were defined as those required to address conditions that are judged to be endangering limb or life. Data on essential vascular services provided at the two centers- (NICVD \& BSHL) through customized vascular surgical teams between April 2020 through June 2021 were analyzed and key outcomes such as mortality and limb salvage rates compared between the two pandemic waves. Chi-square test was used for comparative statistical analysis. A 'P value' of less than 0.05 was considered significant.

Endovascular solutions were given priority over open surgical means whenever deemed appropriate. Local or loco-regional anesthesia were preferred over general anesthesia with endotracheal intubation. Covid-19 was excluded before procedure whenever possible but in the absence of that, every procedure was done considering the case as Covid positive. Standard protective measures including face mask, face shield and personal protective equipment (PPE) were used during procedures. Reverse TranscriptasePolymerase Chain Reaction (RT-PCR) was available as the only testing means for Covid-19. No quick testing was available. Vascular workforce was vaccinated on a priority basis with most members receiving both jabs of their Oxford Astrazeneca vaccine by the early months of 2021.

\section{Results}

Emergency vascular care could be rendered at both facilities in an unhindered manner throughout Covid-19 pandemic. Over the study period, a total of 1792 vascular cases- $90.3 \%$ of which were limb or life threatening issues were addressed by customized vascular 
Table 1: Composition of vascular cases during Covid-19 pandemic.

\begin{tabular}{|c|c|}
\hline Total & 1792 \\
\hline \multirow[t]{5}{*}{ Emergency vascular } & 1112 \\
\hline & Vascular injury (accidental/physical assault): 814 \\
\hline & Acute on chronic limb ischemia: 127 \\
\hline & Arterial pseudoaneurysm/ruptured AVF: 103 \\
\hline & Acute limb ischemia (thrombo-embolic arterial occlusion): 68 \\
\hline \multirow[t]{5}{*}{ Routine vascular } & 399 \\
\hline & Creation of AVF: 173 \\
\hline & Arterial revascularization: 124 \\
\hline & Venous procedures: 77 \\
\hline & Arterio-venous malformation: 25 \\
\hline \multirow[t]{6}{*}{ Vascular catheterization laboratory procedures } & 281 \\
\hline & Percutaneous transluminal angioplasty for CLTI: 155 \\
\hline & Fistuloplasty: 53 \\
\hline & Tunneled catheter insertion: 05 \\
\hline & Renal angio-embbolization: 03 \\
\hline & Diagnostic procedure: 65 \\
\hline
\end{tabular}

AVF: Arterio-Venous Fistula; CLTI: Chronic Limb Threatening Ischemia.

Table 2: Comparative summary of results of vascular procedures during the two pandemic waves.

\begin{tabular}{|c|c|c|c|c|c|}
\hline Disease & Strategy/Procedure & $\begin{array}{c}1^{\text {st }} \text { wave/2nd } \\
\text { wave (N) }\end{array}$ & $\begin{array}{c}\text { Outcome } \\
\text { Procedural } \\
\text { success } \\
\left(1^{\text {st }} \text { wavel }\right. \\
2^{\text {nd }} \text { wave ) } \\
\text { N (\%) }\end{array}$ & $\begin{array}{c}\text { Outcome } \\
\text { Limb salvage } \\
\left(1^{\text {st }} \text { wavel }\right. \\
\left.2^{\text {nd }} \text { wave }\right) \\
\mathrm{N}(\%)\end{array}$ & $\begin{array}{c}\text { Outcome } \\
\text { Mortality } \\
\left(1^{\text {st }} \text { wavel }\right. \\
\left.2^{\text {nd }} \text { wave }\right) \\
\text { N (\%) }\end{array}$ \\
\hline ALI including vascular injury & Repair/embolectomy/amputation & $750 / 132$ & $\begin{array}{c}658(87.7) / 126 \\
(95.4)(P-0.009)\end{array}$ & $\begin{array}{c}652(86.9) / 122 \\
(92.4)(P-0.0759)\end{array}$ & $\begin{array}{c}4(0.5) / 1(0.8) \\
(P-0.0751)\end{array}$ \\
\hline $\begin{array}{l}\text { CLTI with tissue loss } \\
\text { (including acute on chronic) }\end{array}$ & Revascularization with or without amputation & $191 / 60$ & $\begin{array}{c}142(74.3) / 56(93.3) \\
(P-0.001)\end{array}$ & $\begin{array}{c}138(72.6) / 55(91.6) \\
(P-0.001)\end{array}$ & $\begin{array}{l}3(1.6) / 1(1.7) \\
(P-0.9)\end{array}$ \\
\hline HD access creation & $\begin{array}{l}\text { Radio-Cephalic, Brachio-Cephalic, Brachio-Basilic } \\
\text { transposition, tunneled catheter insertion etc. }\end{array}$ & $116 / 62$ & $111(95.6) / 60(96.7)$ & & $0(0) / 0(0)$ \\
\hline HD access care & Fistuloplasty, Repair/ligation of pseudoaneurysm & $89 / 30$ & $85(97.7) / 29(95.5)$ & $88(99 \%) / 29(100)$ & $1(1.1) / 0(0)$ \\
\hline $\begin{array}{l}\text { Aortic/Arterial aneurysm/ } \\
\text { pseudoaneurysm }\end{array}$ & Surgical repair/ligation & $30 / 07$ & $30(100) / 07(100)$ & $30(100) / 07(100)$ & $0(0) / 0(0)$ \\
\hline $\begin{array}{l}\text { Vascular catheterization } \\
\text { laboratory procedures }\end{array}$ & Diagnostic angiogram, PTA & $152 / 71$ & $148(97.3) / 69(97.1)$ & $148(97.3) / 69(97.1)$ & $0(0) / 0(0)$ \\
\hline Venous procedures/AVM & $\begin{array}{c}\text { Varicose vein surgery, RFA, Excision of AVM, } \\
\text { Sclerotherapy }\end{array}$ & $77 / 25$ & $77(100) / 25(100)$ & & $0(0) / 0(0)$ \\
\hline Total & & $1405 / 387$ & & & \\
\hline
\end{tabular}

$1^{\text {st }}$ wave: April 2020 through March- 2021; $2^{\text {nd }}$ wave: April, 2021 through May-2021. ALI: Acute Limb Ischemia; CLTI: Chronic Limb Threatening Ischemia; HD: Hemodialysis; AVM: Arterio-Venous Malformation; PTA: Percutaneous Transluminal Angioplasty; RFA: Radio-Frequency Ablation.

surgical teams. The composition of the cases is illustrated in Table 1.

A wide range of emergencies were handled, the main bulk of which was formed by vascular injuries, acute and chronic limb threatening ischemia and hemodialysis access related issues. Limb salvage was defined as freedom from major amputation. Any amputation above ankle or wrist was considered major.

Overall mortality was $0.6 \%$. Overall limb salvage rates for acute and chronic limb threatening ischemia were $95.3 \%$ and $76.8 \%$, respectively. Limb salvage for acute limb ischemia in the $1^{\text {st }}$ and $2^{\text {nd }}$ pandemic waves were $86.9 \%$ and $92.4 \%$, respectively $(\mathrm{P}=0.0759))$. For Chronic Limb Threatening Ischemia (CLTI) patients, the limb salvage rate was $72.6 \%$ in the $1^{\text {st }}$ wave and $91.6 \%$ in the $2^{\text {nd }}(P=0.001)$. Significantly better outcome was observed in the $2^{\text {nd }}$ wave both in terms of mortality and limb salvage. Results are summarized in Table 2 .
A total of 10,340 patients were given outpatient consultation and 3043 vascular Duplex studies were performed during the pandemic. Telemedicine consultation was provided to 106 patients.

Over the study period, a total of 21 individuals of the vascular workforce at the 2 centers out of a total of 106 contracted Covid-19, 7 of whom were doctors. Most of the infected individuals had mild to moderate symptoms and only 3 required hospital based care. There has been no mortality among the vascular workforce to this point. Comparative analysis of outcome between the 2 waves suggests a better preparedness on the part of the vascular workforce with rate of infection during the $2^{\text {nd }}$ wave being extremely low (Table 3 ).

\section{Discussion}

Covid-19 pandemic has already cost the world more than 4.1 million valuable human lives and the number is increasing every 
Table 3: Comparison of Covid infection among vascular healthcare workers vis a vis other specialties involved in emergency care between 1 st and 2 nd wave.

\begin{tabular}{|c|c|c|c|}
\hline Pandemic wave & Covid Infections (Vascular) N (\%) & Covid Infections (General Surgery) N (\%) & Covid Infections (Cardiology) N (\%) \\
\hline $1^{\text {st }}$ Wave (April, 2020-March, 2021) & $21(19.8)$ & $156(19.1)$ \\
\hline $2^{\text {nd }}$ Wave (April-May, 2021) & $0(0)$ & $32(3.9)$ \\
\hline
\end{tabular}

day. Having fought the initial wave, many countries of the world are now faced with a seemingly more dangerous $2^{\text {nd }}$ and $3^{\text {rd }}$ waves of the pandemic. The Indian subcontinent particularly India became the new epicenter of the $2^{\text {nd }}$ wave in April through May, 2021 as her major cities gasped for oxygen and other treatment essentials. Multiple new variants of the virus have been detected of which the 'Delta variant' has now become a cause for serious concern worldwide. The socioeconomic consequences of the pandemic have already been colossal. The invention of several vaccines has been a tremendous progress in the fight against Covid. Though mass vaccination has been successful in curbing infection [21-23], experts believe that the world might need to live with the virus for at least the next few years. Factors like virus mutation, slow vaccine roll out, social prejudices against vaccine and inequality of vaccine distribution are having negative impact on overall recovery from the pandemic. Covid-19 has placed unprecedented burden on health care system all over the world. The burden is much heavier for countries like Bangladesh where the infrastructure for healthcare delivery is not as strong as in the developed world and there is considerable resource constraints.

Number of practicing vascular surgeons in Bangladesh is only about 30. It was observed that HCW in Bangladesh were contracting infection at a high rate during the $1^{\text {st }}$ wave despite standard protection. This points to the possible pitfalls in the protection gears and practices themselves. Medical grade N95 (NIOSH 1860) masks were not widely available at that time and donning and doffing practices of PPE might have been faulty. It was obvious that HCW were not adequately trained regarding safety protocols. Most of the physicians who have lost their lives to Covid-19 in Bangladesh were aged over 50 and had preexisting conditions. Our customized team approach protects the senior experts by limiting their exposure to possible contamination. Covid-19 infection among vascular workforce has not been particularly high for a specialty involved in emergency services throughout pandemic and no vascular HCW has suffered severe symptoms or death so far. These speak in favor of the effectiveness of our customized team strategy.

We have noticed that despite countrywide lockdown during the early months of the study period, number of vascular emergencies remained high. While this phenomenon is difficult to explain, it may be assumed that these actually were cases redirected from centers that were not operational during the early months of the pandemic and accumulated at centers where services were available. Lockdown as well as the panic of the pandemic also discouraged patient movement causing delayed presentation. All of these had a negative impact on the results particularly limb salvage outcomes. In fact, more than $80 \%$ of our acute limb ischemia patient population arrived after the golden hour. As for chronic limb ischemia patients, over $90 \%$ presented with ulcer or gangrene making some form of amputation inevitable. Rate of major amputation in the $1^{\text {st }}$ wave was higher when compared with the $2^{\text {nd }}$ wave both in acute and chronic ischemia population reflecting the fact that overall preparedness including patient movement and response to vascular emergencies improved considerably in the $2^{\text {nd }}$ wave.

As of July, 2021, Covid-19 situation in Bangladesh continues to deteriorate. Total number of cases has now surpassed 1.2 million with mortality figure exceeding 21,000 [5]. An indication of the deteriorating situation is that NICVD- a dedicated cardiovascular facility was asked to house a 200-bed Covid treatment facility which is now full to its capacity. Bangladesh shares a long and highly porous border with India. Covid situation in some of the Indian states sharing borders with Bangladesh such as West Bengal was grave until recently. Though, the border was officially sealed off for a few weeks in May 2021, a spillover of the Indian wave in Bangladesh is already apparent. Indeed, many Bangladesh districts along the Indian border are still experiencing an alarmingly high rate of infection with test vs case ratios above $50 \%$. Moreover, genome sequencing of virus recently detected in Bangladesh revealed the highly infectious 'Delta variant' in $80 \%$ of the cases which is a clear indication of virus spillover from India. Overall infection in the country has now exceeded 30\% as daily mortality surpasses the 250 mark. Like in the $1^{\text {st }}$ and $2^{\text {nd }}$ waves, constraints in hospital resources including oxygen and ICU beds has once again become obvious. In Bangladesh, hospitals with central oxygen supply is limited and so is the number of ICU beds. Thus, it is only logical to conserve essential resources and working manpower to enhance preparedness for the worse. Our strategy to focus on emergencies, conserve manpower by utilizing the barest minimum for a given task and postponing all non-essential services can be explained in the light of this preparedness.

In Bangladesh, the risk of contracting virus infection among the HCW remains high as the social regulatory measures are not strictly followed. Lack of quick screening for the virus further compromises safety of the caregivers. We believe that the facility of rapid testing for Covid-19 is an important prerequisite for virus containment as well as safer transition to normalcy which has been incorporated in to the national guideline [24]. Rate of Covid infection among the vascular surgeons of Bangladesh is about $20 \%$ which is comparable with that in other communities involved in emergency care during the pandemic. Bangladesh cardiac society reports a total of 132 cases of infection with 6 mortalities among its 750 members over the pandemic year to account for a $17.6 \%$ infection rate and nearly $1 \%$ mortality. Society of surgeons of Bangladesh also reports a similar infection and mortality data. The trend of a lower rate of infection as well as mortality among HCW including the vascular workforce during the $2^{\text {nd }}$ wave has been encouraging. This refers to the fact that HCW in Bangladesh have learned important lessons from the first wave in terms of safety protocol. The positive effect of vaccination in rendering protection has also started to be apparent. The government is pursuing its agenda of mass vaccination which will take a long time in the face of shortages of supply as well as lack of awareness among the common people. At this point, only about 10 million doses of vaccine have been administered $[25,26]$. As we brace for the $3^{\text {rd }}$ wave of the pandemic with plans for a watchful return to the normal vascular activities, we believe that our customized team approach in 
rendering essential services remains relevant for the near future.

\section{Conclusion}

In conclusion, we report our guideline-based customized team strategy in rendering vascular service in Bangladesh during the $1^{\text {st }}$ and $2^{\text {nd }}$ waves of the Covid-19 pandemic. So far, it has stood us in good stead as essential services continue to be delivered negotiating a high volume scenario with satisfactory limb salvage and personnel safety outcomes for the vascular workforce. We believe that these strategies will be key to the continuation of essential vascular services in Bangladesh as the country braces for the $3^{\text {rd }}$ wave of the pandemic.

\section{Declaration}

Statement of informed consent: Informed consent was obtained from all individual participants included in the study

Statement of human and animal rights: The study was approved by the Ethical Review Board of National Institute of Cardiovascular Diseases \& Hospital (NICVD) and Institutional Review Board of Bangladesh Specialized Hospital Limited (BSHL).

\section{References}

1. World Health Organization. Coronavirus disease (Covid-19) pandemic.

2. World Health Organization. Coronavirus disease (Covid-19) pandemic.

3. India Covid.

4. Bangladesh population 2020.

5. Bangladesh Covid.

6. Covid-19: Recommendations for Management of Elective Surgical Procedures.

7. Royal College of Surgeons of England Recovery of surgical services during and after Covid-19. 2020.

8. Søreide K, Hallet J, Matthews B, et al. Immediate and long-term impact of the Covid-19 pandemic on delivery of surgical services. BJS. 2020; 107: 12501261.

9. Kumar S, Shah B, Johari A, et al. Covid-19 Pandemic: Resumption of Orthopedic Care and Medical Education. Indian Journal of Orthopaedics. 2021; 55: 506-515
10. Davenport MS, Bruno MA, Iyer RS, et al. ACR statement of safe resumption of routine radiology care during Covid-19 pandemic. J Am Coll Radiol. 2020; 17: 839-844.

11. Bashar AHM, Hakim ME, Rahman MM, et al. Vascular Surgery Practice Guidelines during Covid-19 Pandemic in a Setting of High Work Volume against Limited Resources: Perspective of a Developing Country. Ann Vasc Surg. 2021; 70: 306-313.

12. Unal EU, Mavioğlu HL, Iscan HZ, et al. Vascular surgery in the Covid-19 pandemic. J Vasc Surg. 2020; 72: 752-754.

13. Melo RG, Pedro LM. Vascular surgery department adjustments in the era of the Covid-19 pandemic. J Vasc Surg. 2020; 72: 375-376.

14. Covid-19 Guidelines for Triage of Vascular Surgery Patients.

15. Lai X, Wang M, Qin C, et al. Coronavirus Disease 2019 (COVID-2019) Infection Among Health Care Workers and Implications for Prevention Measures in a Tertiary Hospital in Wuhan, China. JAMA Network Open. 2020; 3: e209666.

16. Coronavirus (Covid-19): Cases \& Deaths among Healthcare Personnel. 2020.

17. More than $3600 \mathrm{U}$ health workers died in Covid's $1^{\text {st }}$ year. 2021.

18. Fusaroli $P$, Balena $S \&$ Lisotti $A$. On the death of $100+$ Italian doctors from Covid-19. Infection. 2020; 48: 803-804.

19. 747 doctors died of Covid-19 in India: IMA. 2021.

20. Coronavirus: Doctors' mortality rate in Bangladesh 'highest in the world'. 2020.

21. Moghadas SM, Vilches TN, Zhang K, et al. The impact of vaccination on Covid-19 outbreaks in the United States. Clin Infect Dis. 2021.

22. Covid-19 vaccines. 2021

23. Tracking Covid-19 vaccination worldwide. 2021.

24. National Guidelines on Clinical Management of Covid-19. 2020.

25. Covid-19 situation updates. 2021.

26. Bangladesh: WHO Corona virus dashboard. 2021. 